\title{
Celiac disease among at-risk individuals in Saudi Arabia
}

\author{
Mohammad-Ayman A. Safi, PhD (UK).
}

\section{ABSTRACT}

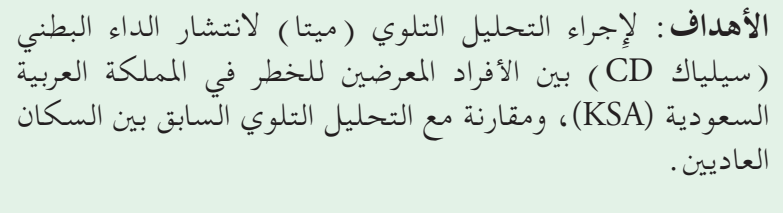

الطريقة : بعد البحث في قواعد البيانات والمجلات ، تم تحليل البيانات

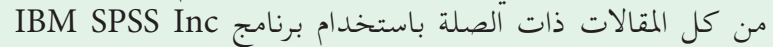
واستخد ام برنامج التحليل التلوي الشامل ( CMA ) .

النتائج: تم العثور على 16 من المقالات ذات الصلة التي تغطي

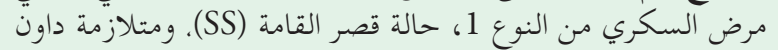

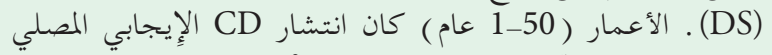

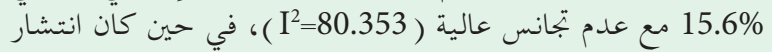

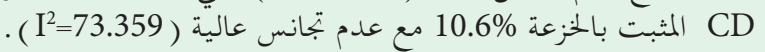

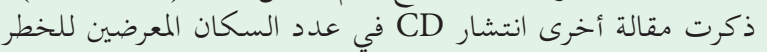

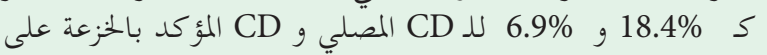

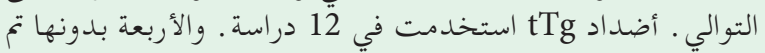

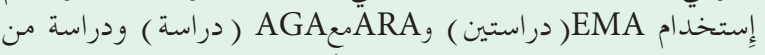
غير تفصيل

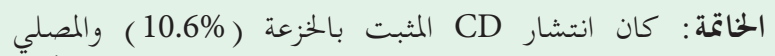

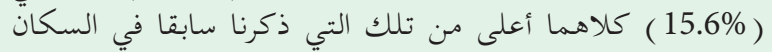

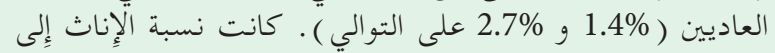

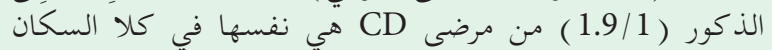

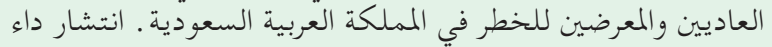

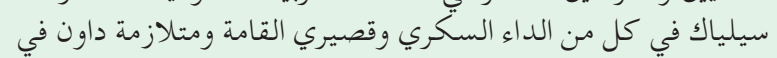

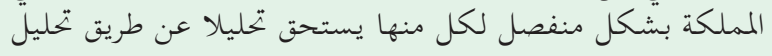
تلوي (ميتا )

Objectives: To perform a meta-analysis for celiac diseases (CD) among at-risk populations in Kingdom of Saudi Arabia (KSA), as well as a comparison with our previously reported meta-analysis in the normal population.

Methods: In March 2018, at King Abdulaziz University, Jeddah, KSA we commenced a retrospective comprehensive database and journal search for $\mathrm{CD}$ among at-risk populations in SA. Data from each of the relevant articles were analyzed using the
Statistical Package for Social Science Version 20 (Armonk, NY: IBM Corp.). and the comprehensive meta-analysis program (CMA). The collected data were part of a retrospective literature review and analysis. Thus, a written ethical approval was not obtained before commencing the study.

Results: Sixteen articles were found covering type-1 diabetes mellitus (DM), short stature (SS), and down syndrome (DS). Ages 1-50 years. The prevalence of seropositive-CD was $15.6 \%$ with high heterogeneity $\left(\mathrm{I}^{2}=80.353\right)$, while prevalence of biopsy-proven CD was $10.6 \%$ with high heterogeneity $\left(\mathrm{I}^{2}=73.359\right)$. Another article reported the CD prevalence in the atrisk population as $18.4 \%$ for the seroprevalence and $6.9 \%$ for the biopsy-proven CD. Anti-transglutaminase (anti-tTG) was used in 12 studies; in the remaining 4 studies (EMA in 2, ARA with AGA in one and no details given in one study).

Conclusion: Both the prevalence of biopsy-proven CD (10.6\%) and seroprevalence (15.6\%) were higher than those we previously reported in the normal population $(1.4 \%$ and $2.7 \%)$. The female-to-male ratio (1.9/1) of $\mathrm{CD}$ patients was the same in normal and at-risk populations in SA. Meta-analysis for prevalence of CD in DM, SS, and DS separately in SA is recommended.

Saudi Med J 2019; Vol. 40 (1): 9-18 doi: 10.15537/smj.2019.1.23892

From the Department of Medical Microbiology and Parasitology, Faculty of Medicine, King Abdulaziz University, Jeddah, Kingdom of Saudi Arabia.

Address correspondence and reprint request to: Dr. Mohammad-Ayman A. Safi, Department of Medical Microbiology and Parasitology, Faculty of Medicine, King Abdulaziz University, Jeddah, Kingdom of Saudi Arabia. E-mail:aymansafi3@hotmail.com

ORCID ID: orcid.org/0000-0002-2217-1332

Celiac disease (CD) is not uncommon in the Kingdom of Saudi Arabia (KSA), with prevalences (in normal populations) of biopsy-proven CD of $1.4 \%$ and seroprevalence of $2.7 \%{ }^{1}$ People at risk for $\mathrm{CD}$ include both genders, of any age or race, having a biological relative with $\mathrm{CD}$, having HLA-DQ2 and 


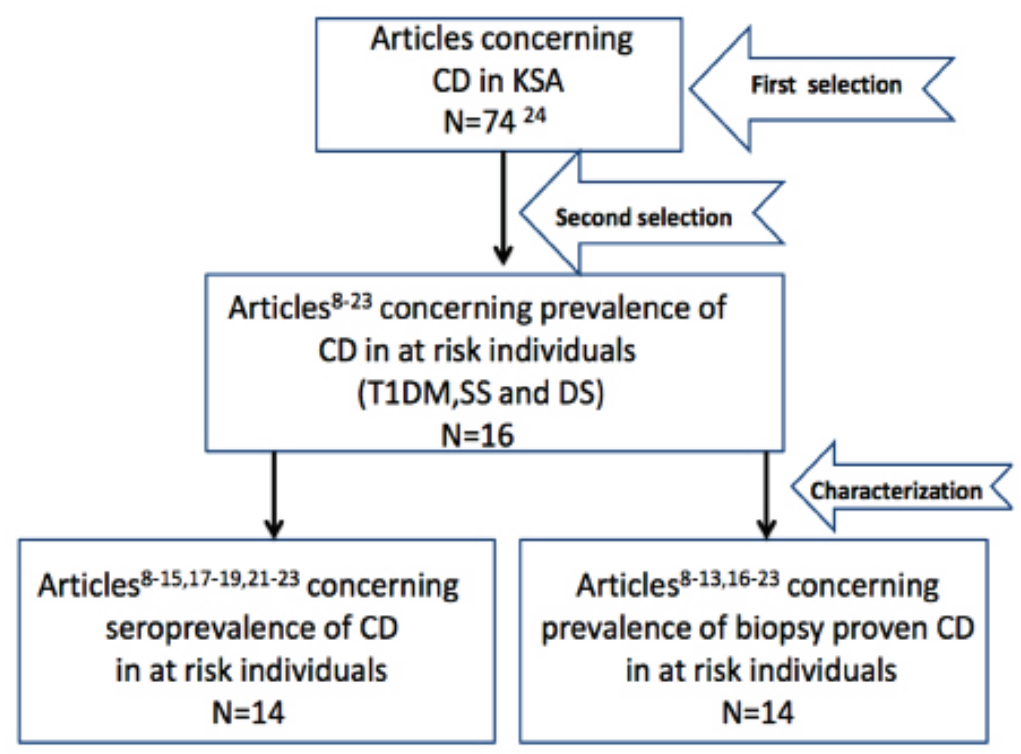

Figure 1 - PRISMA flow-diagram showing the selection process of the pertinent studies. CD- celiac diseases, KSA - Kingdom of Saudi Arabia, T1DM - type 1 diabetes mellitus, SS - short stature, DS - Down syndrome

HLA-DQ8 genes, having some autoimmune diseases or having CD-associated symptoms. ${ }^{2}$ The global prevalence of CD among at-risk groups is 5\% to $10 \%$, which include Down and Turner syndromes, type 1 diabetes, and autoimmune thyroid disease. ${ }^{3}$ In USA, the prevalence of $\mathrm{CD}$ was found to be $2.6 \%$ in seconddegree relatives and $4.5 \%$ in first-degree relatives, with a similar profile in Europe. ${ }^{4}$ Short stature (SS) has also been identified among the at-risk group for CD. Globally, CD in SS ranges from 0.05-59.1\% depending on the region of the study. ${ }^{5,6}$ In KSA, one article reported the prevalence of $\mathrm{CD}$ in the at-risk population as $6.9 \%$ for the biopsy-proven $\mathrm{CD}$ and $18.4 \%$ for the seroprevalence. ${ }^{7}$ The inclusion criteria in that study included chronic diarrhea, abdominal pain or/and T1DM, thyroid disease, failure to thrive (FTT), SS, anemia, and associated neurological diseases; for which the celiac profile was routinely requested. Additionally, 16 articles $^{8-23}$ were found to be concerned specifically with certain at-risk status: T1DM, ${ }^{8-15} \mathrm{SS}^{16-20}$ and Down syndrome. ${ }^{21-23}$ The aim of this study is to perform a meta-analysis for $\mathrm{CD}$ among at-risk populations in $\mathrm{KSA}$, as well as a comparison with our previously reported meta-analysis in the normal population.

Disclosure. Authors have no conflict of interests, and the work was not supported or funded by any drug company.
Methods. Strategy for systematic search and study selection. We retrospectively conducted our systematic review study at King Abdulaziz University, Jeddah, KSA from week 3 of March 2018. A comprehensive database and journal search was used according to the following keywords: "celiac disease in Saudi Arabia", "celiac disease in Saudi children" and "prevalence of celiac disease in Saudi Arabia", followed by a selection process (inclusion/exclusion for the pertinent studies), which was described in detailed in our previous systematic review. ${ }^{24}$ The pooled studies were retrieved via PubMed (US National Library of Medicine, with no specific period), Ovid, EBSCO, and Scholar Google. Few additional related articles were obtained through the library of King Fahd Research Centre, King Abdulaziz University, and directly from the editorial department of the 2 local journals: Saudi Journal of Internal Medicine and Journal of King Abdulaziz University Medical Science. Checking for duplication was carried out between articles via their titles, author(s) and year of publication. Then, 2 selection processes were followed: after matching for duplication, articles that were concerned with CD in KSA were selected (first selection), and their data were recorded using the Statistical Package for Social Science Version 20 (Armonk, NY: IBM Corp.). Articles that are concerned with the at-risk group (T1DM), Down syndrome (DS) and short stature (SS)] were further selected (second 
Table 1 - Characterization of the identified studies on prevalence of celiac disease (CD) in Saudi Arabia.

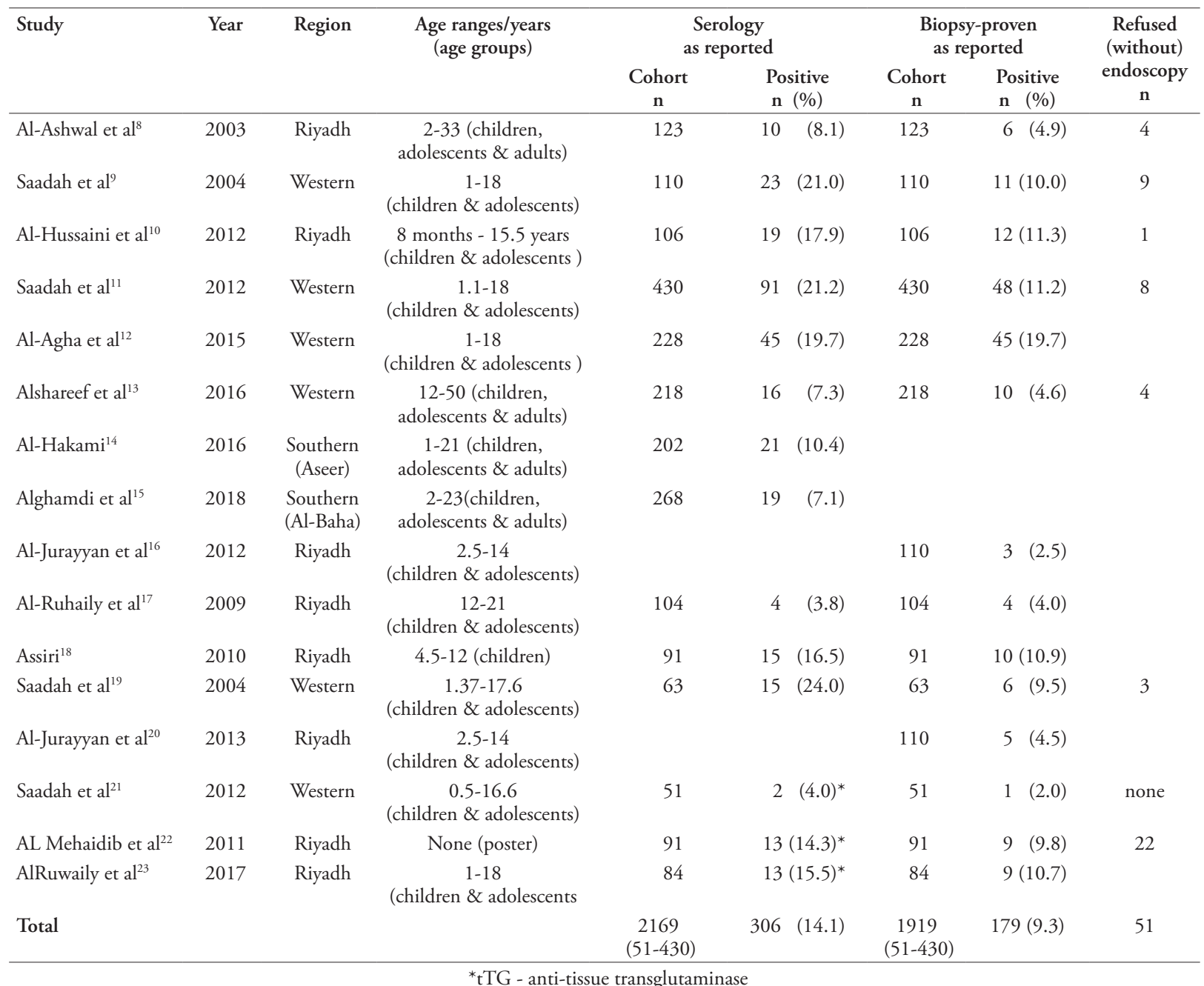

selection) and kept as a separate SPSS file that was used in this study.

Statistical analysis. Data was performed by SPSS (Armonk, NY: IBM Corp.); and by the Comprehensive Meta-analysis program (CMA) (Biostat, USA, Version 3). I squared $\left(\mathrm{I}^{2}\right)$ was used to evaluate heterogeneity. I squared values of $0 \%$ denotes no heterogeneity, 25\% denotes low heterogeneity, $25 \%-49 \%$ denotes moderate heterogeneity and $>50 \%$ denotes high heterogeneity. ${ }^{25}$ The results were illustrated in tabulated form, diagrams, and figures.

Results. Selection and characterization of the pertinent studies (Figure 1 \& Table 1). Seventy-four articles were retrieved (following the matching for duplication and the first selection process) that were concerned with CD in KSA, from which we obtained 16 articles (second selection) concerning CD in at-risk individuals (Figure 1), that are arranged according to the type of the disorder. The data from these studies were recorded using the SPSS Version 20. Characterization of these studies is shown in Table 1 . These studies covered a wide range of ages (1-50 years) and different age groups (Table 1): children and adolescents (10 articles); children and adolescents and adults ( 4 articles); children (one article); no mention (one article). These studies covered 3 regions in KSA (Table 1): Riyadh (8 articles), the western region ( 6 articles) and Southern region ( 2 articles). Table 1 also illustrates the different cohorts and prevalence for both seropositivity and biopsy-proven conditions. 
Table 2 describes the total cohorts, the total number of positivity and rates for both seropositivity and biopsy-proven positivity. The seropositivity total cohort was 2169 (range: 51-430), with a total positivity of 306 and positivity rate of $14.1 \%$ (Table 2 ), while the biopsyproven total cohort was 1919 (range was 51-430), with a total positivity of 179 and positivity rate of $9.3 \%$. Table 2 shows higher rates by meta-analysis (Tables 3 \& 4) for both seropositivity (15.6\%) and biopsy-proven positivity (10.6\%).

Meta-analysis. Meta-analysis was performed using the Comprehensive Meta-analysis program (CMA). Meta analysis was performed for the 14 articles concerning seroprevalence of $\mathrm{CD}$ in at risk individuals [articles 8-15,17-19,21-23], and for the 14 articles concerning prevalence of biopsy-proven CD in at-risk individuals [articles 8-13,16-23]. The meta-analysis of seropositivity prevalence (Tables $3 \& 5$; and Figure $2 \& 3$ ) showed that $\mathrm{CD}$ prevalence (by fixed model) for serologically-proven
$\mathrm{CD}$ (one serology at least) was $15.6 \%(95 \% \mathrm{CI}=14-17.3)$ with high heterogeneity $\left(\mathrm{I}^{2}=80.353\right)$. While metaanalysis for prevalence of biopsy-proven positivity (for 14 articles, by fixed model) (Tables 4 \& 6, Figures 4 \& 5) was $10.6 \%(95 \% \mathrm{CI}=9.5-12.5)$ with high heterogeneity $\left(\mathrm{I}^{2}=73.459\right)$.

Other studies concerning autoimmune diseases versus $C D$ in $K S A$. Five articles ${ }^{26-30}$ concerning autoimmune diseases were not included in the at-risk group of this study. This included two animal studies, two studies of one case each and one study of 42 children ( 24 females; age range between 5-15 years) with juvenile rheumatoid arthritis (JRA). In the latter study, the seroprevalence of CD was $42.8 \%$ (18/42), while the prevalence of biopsy-proven CD was $2.3 \%$ (1/42).

Female to male ratio. Gender information was found in 10 articles (Table 7). The ratio of the total female (120) over the total male (58) was 2/1 (Tables $7 \& 8$ ). Regarding the regions, the western region showed the

Table 2 - Rate of celiac disease in at-risk population; a comparison between the results of the traditional statistical analysis and meta-analysis.

\begin{tabular}{lcccccc}
\hline Variables & $\begin{array}{c}\text { Total } \\
\text { cohort (range) }\end{array}$ & $\begin{array}{c}\text { Serologically } \\
\text { Number of } \\
\text { positive results }\end{array}$ & $\begin{array}{c}\text { Rate } \\
(\%)\end{array}$ & $\begin{array}{c}\text { Cohort } \\
\text { Number of positive } \\
\text { results }\end{array}$ & $\begin{array}{c}\text { Rate } \\
(\%)\end{array}$ \\
\hline Number of studies & 14 & 14 & 14 & 14 & 14 & 14 \\
Duration & $2003-2018$ & $2003-2018$ & $2003-2018$ & $2003-2017$ & $2003-2017$ & $2003-2017$ \\
$\begin{array}{l}\text { Traditional statistical } \\
\text { analysis }\end{array}$ & $2169(51-430)$ & $306(2-91)$ & $306 / 2169(14.1)$ & $1919(51-430)$ & $179(1-48)$ & $179 / 1919(9.3)$ \\
Meta analysis* & $2169(51-430)$ & $306(2-91)$ & $(15.6)$ & $1919(51-430)$ & $179(1-48)$ & $(10.6)$ \\
\hline \multicolumn{7}{c}{$*$ meta-analysis as in Table 3 \& Table 4 } \\
\hline
\end{tabular}

Table 3 - Seropositivity prevalence (by fixed and random models) with the heterogeneity between studies.

\begin{tabular}{|c|c|c|c|c|c|c|c|c|c|c|c|c|c|c|}
\hline \multirow[t]{2}{*}{ Model } & \multirow[t]{2}{*}{$\begin{array}{c}\text { Number of } \\
\text { studies }\end{array}$} & \multicolumn{3}{|c|}{$\begin{array}{l}\text { Effect size and } 95 \% \\
\text { internal }\end{array}$} & \multicolumn{2}{|c|}{$\begin{array}{l}\text { Test of null } \\
\quad(2 \text {-tail })\end{array}$} & \multicolumn{4}{|c|}{ Heterogeneity } & \multicolumn{4}{|c|}{$\mathrm{Tau}^{2}$} \\
\hline & & $\begin{array}{c}\text { Point } \\
\text { estimate }\end{array}$ & $\begin{array}{c}\text { Lower } \\
\text { limit }\end{array}$ & $\begin{array}{c}\text { Upper } \\
\text { limit }\end{array}$ & Z-value & $P$-value & Q-value & $\begin{array}{l}\text { Df } \\
(\mathrm{Q})\end{array}$ & $P$-value & $\mathrm{I}^{2}$ & $\mathrm{Tau}^{2}$ & $\begin{array}{c}\text { Standard } \\
\text { error }\end{array}$ & Variance & Tau \\
\hline Fixed & 14 & 0.156 & 0.140 & 0.173 & -26.952 & 0.000 & 66.167 & 13 & 0.000 & 80.353 & 0.241 & 0.140 & 0.020 & 0.591 \\
\hline Random & 14 & 0.131 & 0.101 & 0.169 & -12.358 & 0.000 & & & & & & & & \\
\hline
\end{tabular}

Table 4 - Prevalence of biopsy-proven positivity (by fixed and random models) with the heterogeneity between studies.

\begin{tabular}{|c|c|c|c|c|c|c|c|c|c|c|c|c|c|c|}
\hline \multirow[t]{2}{*}{ Model } & \multirow[t]{2}{*}{$\begin{array}{c}\text { Number of } \\
\text { studies }\end{array}$} & \multicolumn{3}{|c|}{$\begin{array}{l}\text { Effect size and } \\
95 \% \text { internal }\end{array}$} & \multicolumn{2}{|c|}{$\begin{array}{l}\text { Test of null } \\
\text { (2-tail) }\end{array}$} & \multicolumn{4}{|c|}{ Heterogeneity } & \multicolumn{4}{|c|}{$\mathrm{Tau}^{2}$} \\
\hline & & $\begin{array}{c}\text { Point } \\
\text { estimate }\end{array}$ & $\begin{array}{l}\text { Lower } \\
\text { limit }\end{array}$ & $\begin{array}{l}\text { Upper } \\
\text { limit }\end{array}$ & Z-value & $P$-value & Q-value & $\begin{array}{l}\text { Df } \\
(\mathrm{Q})\end{array}$ & $P$-value & $\mathrm{I}^{2}$ & $\mathrm{Tau}^{2}$ & $\begin{array}{c}\text { Standard } \\
\text { error }\end{array}$ & Variance & Tau \\
\hline Fixed & 14 & 0.106 & 0.092 & 0.122 & -26.734 & 0.000 & 84.981 & 13 & 0.000 & 73.459 & 0.269 & 0.176 & 0.031 & 0.519 \\
\hline Random & 14 & 0.081 & 0.059 & 0.111 & -13.999 & 0.000 & & & & & & & & \\
\hline
\end{tabular}


Table 5 - Data for meta-analysis of the seropositivity prevalence.

\begin{tabular}{lccccc}
\hline Study name & Event rate & Sample size & Event rate & Logit event rate & Standard error \\
\hline Al-Ashwal et al 2003 & 0.081 & 123 & 0.081 & -2.429 & 0.330 \\
Saadah et al 20049 & 0.210 & 110 & 0.210 & -1.325 & 0.234 \\
Al-Hussaini et al 201210 & 0.179 & 106 & 0.179 & -1.523 & 0.253 \\
Saadah et al 201211 & 0.212 & 430 & 0.212 & -1.313 & 0.118 \\
Al-Agha et al 2015 & 0.197 & 228 & 0.197 & -1.405 & 0.167 \\
Alshareef et al 2016 & 0.073 & 218 & 0.073 & -2.541 & 0.260 \\
Al-Hakami 2016 & 0.104 & 202 & 0.104 & -2.154 & 0.230 \\
Alghamdi et al 2018 & 0.071 & 268 & 0.071 & -2.571 & 0.238 \\
Al-Ruhaily \& Malabu 2009 & 0.040 & 104 & 0.040 & -3.178 & 0.500 \\
Assiri 2010 & 0.165 & 91 & 0.165 & -1.621 & 0.282 \\
Saadah et al 2004 & 0.240 & 63 & 0.240 & -1.153 & 0.295 \\
Saadah et al 2012 & 0.040 & 51 & 0.040 & -3.178 & 0.715 \\
AL Mehaidib et al 2011 & 0.142 & 91 & 0.142 & -1.799 & 0.300 \\
AlRuwaily et al 201723 & 0.155 & 84 & 0.155 & -1.696 & 0.301 \\
\hline
\end{tabular}

\begin{tabular}{|c|c|c|c|c|c|c|c|c|c|c|c|c|}
\hline \multirow[t]{2}{*}{ Model } & \multirow[t]{2}{*}{ Study name } & \multicolumn{5}{|c|}{ Statistics for each study } & \multicolumn{4}{|c|}{ Event rate and $95 \% \mathrm{Cl}$} & \multirow{2}{*}{$\begin{array}{l}\text { Weight (Fixed) } \\
\text { Relative weight }\end{array}$} & \multirow{2}{*}{$\begin{array}{l}\text { Weight (Random) } \\
\text { Relative weight }\end{array}$} \\
\hline & & Event rate & Lower limit & Upper limit & ZValue & p-Value & -0.40 & .0 .20 & 0.00 & 0.40 & & \\
\hline & Al-Ashwal $A$ A etal. 2013 & 0.081 & 0.044 & 0.144 & $\cdot 7.349$ & 0.000 & & & $\longrightarrow$ & & 3.601 & $6.68 \mid$ \\
\hline & Saadah, 0 . I etal. 2004 & 0.210 & 0.144 & 0.296 & .5 .660 & 0.000 & & & & & $7.18 \mid$ & $7.91 \mathbf{~}$ \\
\hline & Al.-Hussaini A etal. 2012 & 0.179 & 0.117 & 0.264 & 6.012 & 0.000 & & & & 1 & $6.13 \mid$ & $7.67 \boldsymbol{I}$ \\
\hline & Saadah Ol elal. 2012 & 0.212 & 0.176 & 0.253 & -11.128 & 0.000 & & & & ]$_{1}$ & 28.26 & 9.18 \\
\hline & Al-Agha AE etal. 2015 & 0.197 & 0.150 & 0.254 & .8 .439 & 0.000 & & & & — & 14.19 & 8.71 \\
\hline & Alshareef MA, etal. 2016 & 0.073 & 0.045 & 0.116 & .9 .762 & 0.000 & & & + & & 5.801 & 7.58 \\
\hline & Al.Hakami AM. 2016 & 0.104 & 0.069 & 0.154 & .9 .343 & 0.000 & & & $\rightarrow$ & & $7.40 \mid$ & $7.96 \mathbf{I}$ \\
\hline & Alghamdi RA etal. 2018 & 0.071 & 0.046 & 0.109 & -10.811 & 0.000 & & & + & & 6.95 & $7.87 \mathbf{I}$ \\
\hline & A.-Ruhaily A.D 1, Malabu UH. 2009 & 0.040 & 0.015 & 0.100 & 6.351 & 0.000 & & & $\rightarrow$ & & $1.57 \mid$ & $4.76 i$ \\
\hline & Assiri AM. 2010 & 0.165 & 0.102 & 0.256 & .5 .741 & 0.000 & & & & —- & $4.93 \mid$ & 7.301 \\
\hline & Saadah, 0.1 etal. 2004 & 0.240 & 0.150 & 0.360 & .3 .907 & 0.000 & & & - & & $4.52 i$ & 7.14 \\
\hline & Saadah 0.1 etal. 2012 & 0.040 & 0.010 & 0.145 & .4 .447 & 0.000 & & & + & & $0.77 i$ & $3.11 \mid$ \\
\hline & AL Mehaidib et al. 2011 & 0.142 & 0.084 & 0.230 & .5 .989 & 0.000 & & & $\longrightarrow$ & + & 4.36 & 7.071 \\
\hline & AlRuwaily F etal. 2017 & 0.155 & 0.092 & 0.249 & .5 .625 & 0.000 & & & $\longrightarrow$ & - & 4.331 & 7.05 \\
\hline Fixed & & 0.156 & 0.140 & 0.173 & -26.952 & 0.000 & & & + & & & \\
\hline Random & & 0.131 & 0.101 & 0.169 & .12 .358 & 0.000 & & & + & & & \\
\hline
\end{tabular}

Figure 2 - Seropositivity prevalence (by fixed and random models) with statistics and relative weight for each study.

Model Study name

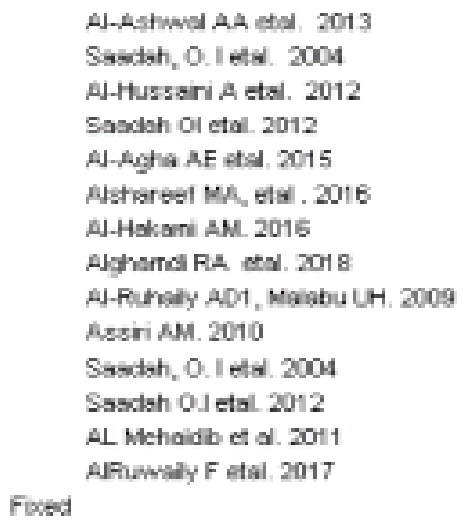

Statistics for eech study

\begin{tabular}{cccrr}
$\begin{array}{c}\text { Event } \\
\text { rate }\end{array}$ & $\begin{array}{c}\text { Lower } \\
\text { limit }\end{array}$ & $\begin{array}{c}\text { Upper } \\
\text { limit }\end{array}$ & \multicolumn{2}{c}{ Z-Vabie p-Value } \\
0.081 & 0.044 & 0.144 & -7.349 & 0.000 \\
0.210 & 0.144 & 0.296 & -5.660 & 0.000 \\
0.179 & 0.117 & 0.264 & -6.012 & 0.000 \\
0.212 & 0.176 & 0.253 & -11.128 & 0.000 \\
0.197 & 0.150 & 0.254 & -8.439 & 0.000 \\
0.073 & 0.045 & 0.116 & -9.762 & 0.000 \\
0.104 & 0.069 & 0.154 & -9.343 & 0.000 \\
0.071 & 0.046 & 0.109 & -10.811 & 0.000 \\
0.040 & 0.015 & 0.100 & -6.351 & 0.000 \\
0.165 & 0.102 & 0.256 & -5.741 & 0.000 \\
0.240 & 0.150 & 0.360 & -3907 & 0.000 \\
0.040 & 0.010 & 0.145 & -4.447 & 0.000 \\
0.142 & 0.094 & 0.230 & -5.969 & 0.000 \\
0.155 & 0.092 & 0.249 & -5.625 & 0.000 \\
0.156 & 0.140 & 0.173 & -26.952 & 0.000
\end{tabular}


Table 6 - Data for meta-analysis of the biopsy-proven positivity.

\begin{tabular}{lccccc}
\hline Study name & $\begin{array}{c}\text { Event } \\
\text { rate }\end{array}$ & $\begin{array}{c}\text { Sample } \\
\text { size }\end{array}$ & $\begin{array}{c}\text { Event } \\
\text { rate }\end{array}$ & $\begin{array}{c}\text { Logit } \\
\text { event } \\
\text { rate }\end{array}$ & $\begin{array}{c}\text { Standard } \\
\text { error }\end{array}$ \\
\hline Al-Ashwal et al 2003 & 0.049 & 123 & 0.049 & -2.966 & 0.418 \\
Saadah et al 20049 & 0.100 & 110 & 0.100 & -2.197 & 0.318 \\
Al-Hussaini et al 2012 & 0.113 & 106 & 0.113 & -2.060 & 0.307 \\
Saadah et al 201211 & 0.112 & 430 & 0.112 & -2.070 & 0.153 \\
Al-Agha et al 2015 & 0.197 & 228 & 0.197 & -1.405 & 0.167 \\
Alshareef et al 2016 & 0.046 & 218 & 0.046 & -3.541 & 0.323 \\
Al-Jurayyan et al 2012 & 0.025 & 110 & 0.025 & -3.032 & 0.611 \\
Al-Ruhaily \& Malabu 2009 & 0.040 & 104 & 0.040 & -3.664 & 0.500 \\
Assiri 2010 & 0.109 & 91 & 0.109 & -2.178 & 0.336 \\
Saadah et al 2004 ${ }^{19}$ & 0.095 & 63 & 0.095 & -2.101 & 0.430 \\
Al-Jurayyan et al 2013 & 0.045 & 110 & 0.045 & -3.254 & 0.460 \\
Saadah et al 2012 & 0.020 & 51 & 0.020 & -3.892 & 1.000 \\
AL Mehaidib et al 2011 22 & 0.098 & 91 & 0.098 & -2.220 & 0.353 \\
AlRuwaily et al 201723 & 0.107 & 84 & 0.107 & -2.122 & 0.353 \\
\hline
\end{tabular}

\begin{tabular}{|c|c|c|c|c|c|c|c|c|c|c|c|c|}
\hline \multirow[t]{2}{*}{ Neast } & \multirow[t]{2}{*}{ stodyrant } & \multicolumn{5}{|c|}{ 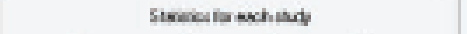 } & \multicolumn{4}{|c|}{ fivelian we E O } & \multirow{2}{*}{ 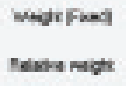 } & \multirow{2}{*}{ 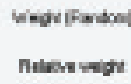 } \\
\hline & & Evarion & Lowe he & Uppe ink & zoven & pirtae & $e x$ & 015 & $\operatorname{tax}$ & $a x$ & & \\
\hline & 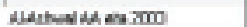 & ores & $a c s$ & 115 & Tाल & $a m x$ & & & $1=$ & & 1ल! & $6 \pi 1$ \\
\hline & 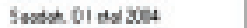 & $0 \times 6$ & 0006 & 112 & 6913 & 0000 & & & $\longrightarrow$ & & tai & 8001 \\
\hline & DHandis new Xol? & Q 113 & $a n g$ & AIE & 6716 & $\mathrm{am}$ & & & $\longrightarrow$ & & $6 \pi$ & $9 \times 1$ \\
\hline & Sandwh 01 atw 300 & 0.12 & aces & 2145 & 1358 & 0000 & & & $\rightarrow$ & & vire & | \\
\hline & Liagade ald $30 t 5$ & $6 \div 9$ & 0.150 & iss & 8434 & 000 & & & & & 248 & 1000 \\
\hline & Divinilith nes Xat & out & anes & 100 & $\sin$ & $\mathrm{ami}$ & & & + & & $6 \infty 1$ & 901 \\
\hline & Ablur on K Mats ans & ora & $a v e$ & 1000 & $5 x$ & 000 & & & 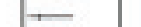 & & $1 \pi 0$ & $4 \pi \hat{i}$ \\
\hline & 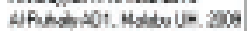 & $0(4)$ & 0005 & $1+100$ & 698 & 000 & & & $\longrightarrow$ & & 254 & $5 \pi 1$ \\
\hline & Inaks 2010 & ong & 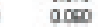 & a1si & EUE & $a \infty x$ & & & - & & $=1$ & $79 i$ \\
\hline & 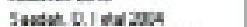 & $0 \mathrm{set}$ & ouns & $11 \%$ & 5245 & 000 & & & $\longrightarrow$ & & $14 i$ & 5า i \\
\hline & dilamon kaveds $30 \mathrm{~s}$ & 065 & 000 & atea & 650. & 000 & & & - & & ial & 6231 \\
\hline & Sactiv al asd $\mathrm{xr} 12$ & orm & $a 00$ & 2127 & $2 x$ & $000 x$ & & & . & & Еल| & $2 \times i$ \\
\hline & 4. Motodte it al Jon & ors & ausz & $11 \times 0$ & $5=0$ & owe & & & $\longrightarrow$ & & $=171$ & 网 \\
\hline & 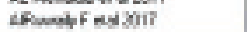 & $6=0$ & 000 & $=169$ & 611 & $a \omega$ & & & $\longrightarrow$ & & stei & $7 \mathrm{ea} i$ \\
\hline nas & & $07 x$ & 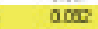 & 2122 & $x 7 y$ & $a D x$ & & & + & & & \\
\hline Fondon & & ox & aubs & 1171 & 1390 & owox & & & $\rightarrow$ & & & \\
\hline
\end{tabular}

Figure 4 - Prevalence of biopsy-proven celiac diseases (by fixed and random models) with statistics and relative weight for each study.

Model study name

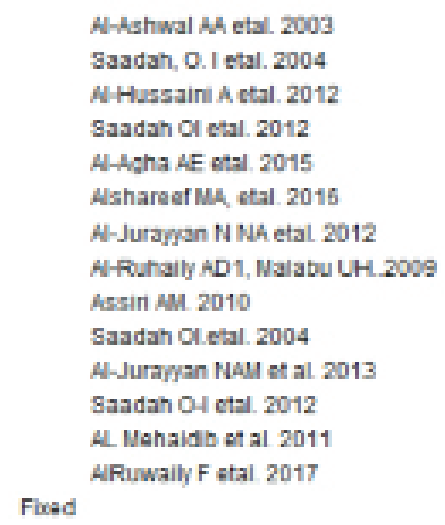

Statistics for each study

\begin{tabular}{|c|c|c|c|c|}
\hline $\begin{array}{l}\text { Event } \\
\text { rate }\end{array}$ & $\begin{array}{l}\text { Lewer } \\
\text { limnit }\end{array}$ & $\begin{array}{l}\text { Upoer } \\
\text { Imit }\end{array}$ & Z-Value & p-Value \\
\hline 0.049 & 0.022 & 0.105 & -7.100 & 0.000 \\
\hline 0.100 & 0.080 & 0.172 & -6.913 & 0.000 \\
\hline 0.113 & 0.065 & 0.189 & -6.716 & 0.000 \\
\hline 0.112 & 0.085 & 0.145 & -13540 & 0.000 \\
\hline 0.197 & 0.150 & 0.254 & -8430 & 0000 \\
\hline 0.046 & 0.005 & 0.083 & .9378 & 0.000 \\
\hline 0.025 & 0.008 & 0.078 & -5999 & 0.000 \\
\hline 0.040 & 0.015 & 0.100 & -6.351 & 0.000 \\
\hline 0.109 & 0.060 & 0.191 & -6246 & 0.000 \\
\hline 0.095 & 0.043 & 0.196 & -5246 & 0.000 \\
\hline 0.045 & 0.019 & 0.104 & 6642 & 0.000 \\
\hline 0.020 & 0.003 & 0127 & 36en & 0.000 \\
\hline 0.098 & 0.052 & 0170 & 6.205 & 0.000 \\
\hline 0.107 & $005 ?$ & 0.123 & -6011 & 0000 \\
\hline 0.106 & 0.092 & 0.122 & -26734 & 0000 \\
\hline
\end{tabular}


highest $\mathrm{F} / \mathrm{M}$ ratio $(24 / 8=3 / 1)$ followed by the southern region $(35 / 16=2.1 / 1)$ and Riyadh $(61 / 34=1.8 / 1)$ (Table 8).

Duration span. The included 16 studies were published between 2004 and 2018, covering a long period from 1990 until 2014, with 5 retrospective studies without year limitation..$^{911,19,22}$

The pattern of serology and biopsy. In the 16 included studies, anti-t TG was used in 12 studies (anti-t TG alone in 5 studies; with EMA in 3; with AGA in one; with EMA and AGA in 2; with EMA and AGA and ARA in one), and biopsy was used in 14 studies. Without anti-tTG: EMA was used in 2 studies: ARA with AGA (one study) and one study without details (Table 7).

Conditions of the CD at-risk studies and their distribution according to regions in KSA. The 16 studies are related to 3 regions (Riyadh, western and southern). Riyadh showed the highest at-risk studies (8 studies) followed by the western region (6 studies) and southern region (2 studies) (Table 8 ). Riyadh had the most studies for SS and DS (4 out of 5 studies for SS and 2 out of 3 for DS), followed by the western region (one out of 5 for SS and one out of 3 for DS), while the western region showed the highest for T1DM studies ( 4 out of 8 studies) followed by Riyadh and the southern region (2 studies each).

Prevalence of $C D$ in different at-risk conditions (T1DM, DS, SS) in KSA. The reported biopsy-proven prevalence showed considerable range variations among studies of the 3 conditions: $7.6-19.7 \%$ for DM, $2-10.7 \%$ for DS, and 2.5-10.9\% for SS (Table 9).

Table 8 - Conditions of the celiac disease at-risk studies and their distribution according to regions in Saudi Arabia.

\begin{tabular}{|c|c|c|c|c|}
\hline Region & Condition & Reference & $\begin{array}{c}\text { Number of } \\
\text { studies }\end{array}$ & Female/Male \\
\hline Riyadh & $\begin{array}{l}\text { T1DM } \\
\text { SS } \\
\text { DS }\end{array}$ & $\begin{array}{c}8,10 \\
16-18,20 \\
22,23\end{array}$ & $\begin{array}{l}2 \\
4 \\
2\end{array}$ & $61 / 34=1.8 / 1$ \\
\hline Western & $\begin{array}{l}\text { T1DM } \\
\text { SS } \\
\text { DS }\end{array}$ & $\begin{array}{c}9,11-13 \\
19 \\
21\end{array}$ & $\begin{array}{l}4 \\
1 \\
1\end{array}$ & $24 / 8=3 / 1$ \\
\hline Southern & T1DM & 14,15 & 2 & $35 / 16=2.1 / 1$ \\
\hline Total & & & 16 & $120 / 58=2 / 1$ \\
\hline
\end{tabular}

Table 7 - Pattern of serology and biopsy in the identified studies on prevalence of at-risk individuals for celiac disease (CD) in Saudi Arabia.

\begin{tabular}{|c|c|c|c|c|c|c|c|c|}
\hline Study & Year & Serology & tTG* & Biopsy** & Female $^{\ddagger}$ & Male $^{\ddagger}$ & Age range (years) & Period \\
\hline Al-Ashwal et al ${ }^{8}$ & 2003 & AGA, ARA & No & Yes & 2 & 8 & $2-33$ & $1995-1996$ \\
\hline Saadah et al ${ }^{9}$ & 2004 & tTG, AGA & Yes & Yes & NS & NS & $1-18$ & Retrospectively \\
\hline Al-Hussaini et $\mathrm{al}^{10}$ & 2012 & $\begin{array}{l}\text { tTG-IgA, } \\
\text { EmA-IgA }\end{array}$ & Yes & Yes & 11 & 1 & $\begin{array}{c}8 \text { months to } \\
15.5 \text { years }\end{array}$ & Prospective; $2008-2010$ \\
\hline Saadah et $\mathrm{al}^{11}$ & 2012 & tTG-IgA & Yes & Yes & NS & NS & $1.1-18$ & $\begin{array}{l}\text { Retrospective hospital record-based } \\
\text { study of all children and adolescents } \\
\text { with T1DM who were screened for CD }\end{array}$ \\
\hline Al-Agha et $\mathrm{al}^{12}$ & 2015 & tTG-IgA & Yes & Yes & NS & NS & $1-18$ & January 2013 - January 2014 \\
\hline Alshareef et $\mathrm{al}^{13}$ & 2016 & $\mathrm{tTG}$ & Yes & Yes & 12 & 4 & $12-50$ & January 2008 - June 2009 \\
\hline $\mathrm{Al}-\mathrm{Hakami}^{14}$ & 2016 & tTG, EmA & Yes & No & 24 & 8 & $1-21$ & March 2013 - June 2014 \\
\hline Alghamdi et a ${ }^{15}$ & 2018 & $\mathrm{tTG}$ & Yes & No & 11 & 8 & $2-23$ & Retrospective record base \\
\hline Al-Jurayyan et $\mathrm{al}^{16}$ & 2012 & $\begin{array}{c}\text { Celiac } \\
\text { screening }\end{array}$ & $?$ & Yes & 2 & 8 & $2.5-14$ & January 1990 - December 2009 \\
\hline Al-Ruhaily et al ${ }^{17}$ & 2009 & $\mathrm{EmA}$ & No & Yes & NS & NS & $12-21$ & January 1997 - December 2006 \\
\hline Assiri ${ }^{18}$ & 2010 & $\begin{array}{l}\text { tTG-IgA, } \\
\text { EmA-IgA }\end{array}$ & Yes & Yes & 11 & 1 & $4.5-12$ & August 2002 - December 2008 \\
\hline Saadah et $\mathrm{al}^{19}$ & 2004 & tTG, AGA & Yes & Yes & NS & NS & $1.37-17.6$ & Retrospectively \\
\hline Al-Jurayyan et $\mathrm{al}^{20}$ & 2013 & EMA & No & Yes & NS & NS & $2.5-14$ & January 1990 - December 2009 \\
\hline Saadah et $\mathrm{al}^{21}$ & 2012 & tTG-IgA & Yes & Yes & 12 & 4 & $0.5-16.6$ & January 2007 - August 2011 \\
\hline AL Mehaidib et $\mathrm{a}^{22}$ & 2011 & $\begin{array}{l}\text { tTG, Ema, } \\
\text { AGA, ARA }\end{array}$ & Yes & Yes & 24 & 8 & None (poster) & Retrospectively \\
\hline AlRuwaily et al ${ }^{23}$ & 2017 & $\begin{array}{c}\text { tTG, EMA, } \\
\text { AGA }\end{array}$ & Yes & Yes & 11 & 8 & $1-18$ & Retrospectively \\
\hline Total & & & & & $120^{\ddagger}$ & $58^{\ddagger}$ & & \\
\hline
\end{tabular}


Table 9 - Rate of celiac disease in different at-risk populations (total at-risk, type1 diabetes mellitus (DM1), short stature(SS) and Down syndrome (DS).

\begin{tabular}{lcccccccc}
\hline & \multicolumn{4}{c}{ Serologically } & \multicolumn{3}{c}{ Biopsy -proven } \\
& At risk & DM1 & SS & DS & At risk & DM1 & SS & DS \\
\hline Number of studies & 14 & 8 & 3 & 3 & 14 & 6 & 5 & 3 \\
Duration & $2003-2018$ & $2003-2018$ & $2004-2010$ & $2004-2010$ & $2011-2017$ & $2003-2016$ & $2004-2013$ & $2011-2017$ \\
Cohort & 2169 & 1685 & 258 & 226 & 1919 & 1215 & 478 & 226 \\
positive & 306 & 244 & 34 & 28 & 179 & 132 & 28 & 19 \\
Rate\% & $306 / 2169$ & $244 / 1685$ & $34 / 258$ & $28 / 226$ & $179 / 1919$ & $132 / 1215$ & $28 / 478$ & $19 / 226$ \\
& $=14.1 \%$ & $=14.4 \%$ & $=13.17 \%$ & $=12.38 \%$ & $=9.3 \%$ & $=10.8 \%$ & $=5.85 \%$ & $=8.4 \%$ \\
\hline
\end{tabular}

However, the calculated biopsy-proven prevalence of CD was highest in DM (10.8 [132/1215]), followed by DS (8.4\% [19/226]) and lowest in SS (5.85 [28/478]).

Discussion. Celiac disease is more common in at-risk people, such as having a biological relative with CD, having HLA-DQ2 and HLA-DQ8 genes, having $\mathrm{CD}$ symptoms or having some autoimmune diseases. ${ }^{2}$ For genetic-based CD, there is an increased risk (3-6\%) for developing CD among those with a relative having CD2. Likewise, having HLA-DQ2 and HLA-DQ8 haplotypes is associated with increased risk for developing CD, while their absence excludes the possibility of CD31. Globally, approximately $95 \%$ of people with CD have the HLA-DQ2 gene, and most of the remaining $5 \%$ have the HLA-DQ8 gene. ${ }^{2}$ However, it is reported that HLA-DQ2 and HLA-DQ8 were present in $98.6 \%$ of patients with CD, and in $40 \%$ of the global general population who were without a diagnosis of CD. ${ }^{32}$ However, HLA-DQ in KSA has been recently reported to be one of the highest frequencies $(52.7 \%)$ among healthy general populations worldwide. ${ }^{33}$ Having the HLA-DQ gene indicates that the person is at risk for developing $\mathrm{CD}$, but does not indicate a definite CD; thus, a positive HLA-DQ test should be followed by CD profile tests (blood and histology) for the determination of presence or absence of CD. ${ }^{34}$

Screening of CD is becoming increasingly common in first-degree relatives and patients with T1DM. ${ }^{35,36}$ The presence of an autoimmune disease (such as CD) increases the likelihood of developing other autoimmune diseases, such as T1DM, ${ }^{37,38}$ autoimmune thyroid disease, ${ }^{39,40}$ autoimmune hepatitis ${ }^{41}$ and DS; ${ }^{42}$ thus, these conditions, in addition to first-degree relatives, are usually termed as high-prevalence or at-risk groups, in which screening of CD (in contrast to the general population) is considered to have a favourable cost-benefit ratio. ${ }^{43}$ The current study represents the first and only meta-analysis concerning the prevalence of CD in at-risk individuals in KSA. This meta-analysis included more than 2000 at-risk individuals from 16 studies in KSA. The 16 articles were covering the at-risk population (T1DM, SS and DSe). Two articles of them were without endoscopy results, and the other 2 only showed the biopsy-confirmed results. Thus, both seroprevalence CD and prevalence of biopsy-confirmed CD were calculated in 14 articles each. By metaanalysis, the prevalence of biopsy-proven CD (10.6\%) and seroprevalence $(15.6 \%)$ were both high, with high heterogeneity $\left(\mathrm{I}^{2}=73.459\right.$ and $\left.\mathrm{I}^{2}=80.353\right)$; while with a traditional statistics approach by SPSS analysis, 14.1\% had seropositivity and 9.3\% had biopsy-proven CD. Meta-analysis allows a more objective appraisal than the traditional statistical analysis, since it takes into account the relative weight of each involved study and, thus, provides a more precise estimate, in addition to the heterogeneity between the results of individual studies. ${ }^{44}$ The global prevalence of CD among at-risk groups (Down and Turner syndromes, type 1 diabetes, and autoimmune thyroid disease) have been reported to range from $5 \%$ to $10 \% .^{3}$ In KSA, one article reported the prevalence in the at-risk population as $18.4 \%$ and $6.9 \%$ for the seroprevalence and biopsy-proven CD respectively. ${ }^{7}$

Other articles 26-30 were found concerning autoimmune diseases. These articles include two animal studies, in addition to one case study ( 2 articles). One study addressed 42 children (24 females; age ranged between 5-15 years) with juvenile rheumatoid arthritis (JRA). The latter study showed a considerable difference between seroprevalence of CD (42.8\%) and the prevalence of biopsy-proven CD (2.3\%) and was not involved in the current meta-analysis. In comparison with the CD prevalence in normal populations in KSA, ${ }^{1}$ both the prevalence of biopsy-proven CD (10.6\%) and seroprevalence $(15.6 \%)$ were higher than that in the normal population $(1.4 \%$ and $2.7 \%)$. Similar to the normal population ${ }^{1}$ the total number of the $\mathrm{CD}$ females among the at-risk population was twice as much as 
the CD males (1.9/1). The reported prevalence in the current study showed that biopsy-proven prevalence of CD was highest in DM (10.8 [132/1215]), followed by DS (8.4\% [19/226]) and lowest in SS (5.85 [28/478]); this is in contrast to the report of Costa Gomes et $\mathrm{al}^{42}$ from Brazil that biopsy-proven $\mathrm{CD}$ prevalence in DS is almost three-times higher than that in DM [DM1=5/111 (4.5\%), DS=10/77 (13.0\%)].

One limitation is the absence of uniform performance of small intestine biopsies in seropositive patients, as (in seven studies) 51 out of 134 seropositive patients (38\%) refused endoscopy; another limitation is the lack of gender ratio in some studies.

In conclusion, concerning CD in at-risk individuals in $\mathrm{KSA}$, both the prevalence of biopsy-proven $\mathrm{CD}$ (10.6\%) and seroprevalence (15.6\%) were high, with high heterogeneity $\left(\mathrm{I}^{2}=73.459\right.$ and $\left.\mathrm{I}^{2}=90.353\right)$ between studies; and both are higher than those in the normal population (1.4\% and $2.7 \%)$. The female-to-male ratio (1.9/1) of CD patients was the same in the normal and at-risk populations in KSA.

\section{References}

1. Safi MAA. Prevalence of Celiac disease in Saudi Arabia: Metaanalysis. Global Vaccines and Immunology 2018; 3: 1-6.

2. Fasano A, Berti I, Gerarduzzi T, Not T, Colletti RB, Drago S, et al. Prevalence of celiac disease in at-risk and not-at-risk groups in the United States: a large multicenter study. Arch Intern Med 2003; 163: 286-292.

3. Barker JM, Liu E. Celiac disease: pathophysiology, clinical manifestations, and associated autoimmune conditions. $A d v$ Pediatr 2008; 55: 349-365.

4. Gujral N, Freeman HJ, Thomson AB. Celiac disease: prevalence, diagnosis, pathogenesis and treatment. World J Gastroenterol 2012; 18: 6036-6059.

5. Bonamico M, Sciré G, Mariani P, Pasquino AM, Triglione P, Scaccia $S$, et al. Short stature as the primary manifestation of monosymptomatic celiac disease. J Pediatr Gastroenterol Nutr 1992; 14: 12-16.

6. Rossi TM, Albini CH, Kumar V. Incidence of celiac disease identified by the presence of serum endomysial antibodies in children with chronic diarrhea, short stature, or insulindependent diabetes mellitus. J Pediatr 1993; 123: 262-264.

7. Al-Hakami AM. Seroprevalence of coeliac disease in at-risk subjects at the main tertiary hospital, southwest of Saudi Arabia. Arab J Gastroenterol 2016; 17: 41-44.

8. Al-Ashwal AA, Shabib SM, Sakati NA, Attia NA. Prevalence and characteristics of celiac disease in type I diabetes mellitus in Saudi Arabia. Saudi Med J 2003; 24: 1113-1115.

9. Saadah OI, Al Agha AE, Albokhari SM, Al Mughales JA. P0408 prevalence of celiac disease in saudi children with type 1 diabetes mellitus. Journal of Pediatric Gastroenterology and Nutrition 2004; 39: S211.

10. Al-Hussaini A, Sulaiman N, Al-Zahrani M, Alenizi A, El Haj I. High prevalence of celiac disease among Saudi children with type 1 diabetes: a prospective cross-sectional study. $B M C$ Gastroenterol 2012; 12: 180.
11. Saadah OI, Al-Agha AE, Al Nahdi HM, Bokhary RY, Bin Talib YY, Al-Mughales JA, et al. Prevalence of celiac disease in children with type 1 diabetes mellitus screened by anti-tissue transglutaminase antibody from Western Saudi Arabia. Saudi Med J 2012; 33: 541-546.

12. Al-Agha AE, Alafif MM, Abd-Elhameed IA. Glycemic control, complications, and associated autoimmune diseases in children and adolescents with type 1 diabetes in Jeddah, Saudi Arabia. Saudi Med J 2015; 36: 26-31.

13. Alshareef MA, Aljabri KS, Bokhari SA, Al Jiffri AM, Abu Elsaoud HM, Akl AF, et al. The prevalence of celiac disease in saudi patients with type 1 diabetes mellitus: Cross sectional study. Int J Diabetes Metab Disord 2016; 1: 1-4.

14. Al-Hakami AM. Pattern of thyroid, celiac, and anti-cyclic citrullinated peptide autoantibodies coexistence with type 1 diabetes mellitus in patients from Southwestern Saudi Arabia. Saudi Med J 2016; 37: 386-391.

15. Alghamdi RA, Alghamdi AH, Fureeh AA. Sero-prevalence of celiac disease among symptom-free type 1 diabetes mellitus in Al-Baha Region, Saudi Arabia. IOSR Journal of Pharmacy and Biological Sciences (IOSR-JPBS) 2018; 13: 22-26.

16. Al-Jurayyan N NA, Mohamed SH, Al Otaibi HM, Al Issa ST, Omer HG. Short stature in children: Pattern and frequency in a pediatric clinic, Riyadh, Saudi Arabia. Sudan J Paediatr 2012; 12: 79-83.

17. Al-Ruhaily AD, Malabu UH. Short stature in Saudi Arabia: etiologic profile in adult endocrine clinic. Niger J Med 2009; 18: 268-271.

18. Assiri AM. Isolated short stature as a presentation of celiac disease in Saudi children. Pediatr Rep 2010; 2: e4.

19. Saadah OI, Al Agha AE, Albokhari SM, Al Mughales JA. P0405 screening of saudi children with short stature for celiac disease. Journal of Pediatric Gastroenterology and Nutrition 2004; 39: S210.

20. Al-Jurayyan N AM, Al Nemri AMH, Al Jurayyan ANA, Assiri AMA. Celiac disease in children with short stature: A hospital based study. Journal of Taibah University Medical Sciences 2013; 8: 93-96.

21. Saadah OI, Al-Aama JY, Alaifan MA, Bin Talib YY, Al-Mughales JA. Prevalence of celiac disease in children with Down syndrome screened by anti-tissue transglutaminase antibodies. Saudi Med J 2012; 33: 208-210.

22. Al Mehaidib A, Kattan H, AL Rrowaily F, AL Dekhail W. Prevalence of celiac disease in Saudi children with Down syndrome: a retrospective study. J Gastroenterol Hepatol 2011; 26 (Suppl 5): 84.

23. AlRuwaily F, Kattan HA, AlMehaidib AM, AlDekhail W. Prevalence of celiac disease in Saudi children with Down syndrome: A retrospective study. International Journal of Pediatrics and Adolescent Medicine 2017; 4: 51-53.

24. Safi MAA. Safi HMA. Celiac disease and its serological pattern in Saudi Arabia; A systematic review. Global Vaccines and Immunology 2018; 3: 1-15.

25. Singh P, Arora A, Strand TA, Leffler DA, Catassi C, Green PH, et al. Global prevalence of celiac disease: systematic review and meta-analysis. Clin Gastroenterol Hepatol 2018; 16: 823-836.

26. Bin-Abbas BS, Faiyaz-Ul-Haque M, Al-Fares AH, Al-Gazlan SS, Bhuiyan JA, Al-Muhsen SZ. Autoimmune polyglandular syndrome type 1 in Saudi children. Saudi Med J 2010; 31: 788-792. 
27. Al-Mayouf SM, Al-Mehaidib AI, Alkaff MA. The significance of elevated serologic markers of celiac disease in children with juvenile rheumatoid arthritis. Saudi J Gastroenterol 2003; 9: 75-78.

28. Vangone A, Abdel-Azeim S, Caputo I, Sblattero D, Di Niro $\mathrm{R}$, Cavallo L, et al. Structural basis for the recognition in an idiotype-anti-idiotype antibody complex related to celiac disease. PLoS One 2014; 9: e102839.

29. Alzahrani AS, Al Sheef M. Severe primary hyperparathyroidism masked by asymptomatic celiac disease. Endocr Pract 2008; 14 : 347-350.

30. Selim ME, Al-Ayadhi LY. Possible ameliorative effect of breastfeeding and the uptake of human colostrum against coeliac disease in autistic rats. World J Gastroenterol 2013; 19: 3281-3290.

31. Megiorni F, Pizzuti A. HLA-DQA1 and HLA-DQB1 in Celiac disease predisposition: practical implications of the HLA molecular typing. J Biomed Sci 2012; 19: 88.

32. Cecilio LA, Bonatto MW. [The prevalence of HLA DQ2 and DQ8 in patients with celiac disease, in family and in general population]. Arq Bras Cir Dig 2015; 28: 183-185. Portuguese

33. Al-Hussaini A, Alharthi H, Osman A, Eltayeb-Elsheikh N, Chentoufi A. Genetic susceptibility for celiac disease is highly prevalent in the Saudi population. Saudi J Gastroenterol 2018; 24: 268-273.

34. Beyond Celiac. Celiac Disease: Who is at Risk? [Updated 2018, Accessed 2018 May 17]. Available from: https://www. beyondceliac.org/celiac-disease/risk-factors/

35. Dubé C, Rostom A, Sy R, Cranney A, Saloojee N, Garritty $\mathrm{C}$, et al. The prevalence of celiac disease in average-risk and at-risk Western European populations: a systematic review. Gastroenterology 2005; 128: S57-S67.

36. Ludvigsson JF, Bai JC, Biagi F, Card TR, Ciacci C, Ciclitira PJ, et al. Diagnosis and management of adult coeliac disease: guidelines from the British Society of Gastroenterology. Gut 2014; 63: 1210-1228.
37. Fröhlich-Reiterer EE, Hofer S, Kaspers S, Herbst A, Kordonouri $\mathrm{O}$, Schwarz HP, et al. Screening frequency for celiac disease and autoimmune thyroiditis in children and adolescents with type 1 diabetes mellitus--data from a German/Austrian multicentre survey. Pediatr Diabetes 2008; 9: 546-553.

38. Uibo O, Heilman K, Rägo T, Shor R, Paal M, Metsküla K, et al. Symptomless celiac disease in type 1 diabetes: 12-year experience in Estonia. Pediatr Int 2010; 52: 230-233.

39. Mainardi E, Montanelli A, Dotti M, Nano R, Moscato G. Thyroid-related autoantibodies and celiac disease: a role for a gluten-free diet? J Clin Gastroenterol 2002; 35: 245-248.

40. Meloni GF, Tomasi PA, Bertoncelli A, Fanciulli G, Delitala G, Meloni T. Prevalence of silent celiac disease in patients with autoimmune thyroiditis from Northern Sardinia. J Endocrinol Invest 2001; 24: 298-302.

41. Villalta D, Girolami D, Bidoli E, Bizzaro N, Tampoia $\mathrm{M}$, Liguori $\mathrm{M}$, et al. High prevalence of celiac disease in autoimmune hepatitis detected by anti-tissue tranglutaminase autoantibodies. J Clin Lab Anal 2005; 19: 6-10.

42. Costa Gomes R, Cerqueira Maia J, Fernando Arrais R, André Nunes Jatobá C, Auxiliadora Carvalho Rocha M, Edinilma Felinto Brito $\mathrm{M}$, et al. The celiac iceberg: from the clinical spectrum to serology and histopathology in children and adolescents with type 1 diabetes mellitus and Down syndrome. Scand J Gastroenterol 2016; 51: 178-185.

43. Aggarwal S, Lebwohl B, Green PH. Screening for celiac disease in average-risk and high-risk populations. Therap $A d v$ Gastroenterol 2012; 5: 37-47.

44. Egger M, Smith GD. Meta-analysis. Potentials and promise. BMJ 1997; 315: 1371-1374. 